\title{
Editorial: The Top 10 Questions in Primatology
}

\author{
Joanna M. Setchell
}

Received: 15 May 2013 / Accepted: 15 May 2013 /Published online: 3 July 2013

(C) Springer Science+Business Media New York 2013

In 2012 I launched a search for the top 10 questions that remain unanswered in primatology, in my roles as Editor-in-Chief of this journal and Vice President for Research of the International Primatological Society (IPS). This initiative stemmed from a Nature News item reporting on searches for the most pressing questions in social science, conducted independently by Harvard and the U.S. National Science Foundation (Giles 2011). The "top 10" approach used by Harvard was, in turn, inspired by a 1900 list of major unsolved questions in mathematics, compiled by David Hilbert (Giles 2011). Similar exercises (horizon scans) have been used to identify critical knowledge gaps in science (Kennedy and Norman 2005), emerging global conservation issues (Sutherland et al. 2009, 2010, 2011, 2012, 2013a), and the major unanswered questions in basic ecology (Sutherland et al. 2013b).

The aim of the "Top 10 Questions in Primatology" is to set the agenda for the future of primatology. I launched the search in an editorial in the International Journal of Primatology (IJP) in 2012 (Setchell 2012), announced it at the XXIV IPS Congress in Cancun in 2012, and advertised it on the IJP website. I followed this up with an e-mailed invitation to the IPS membership to contribute to the search via a dedicated e-mail account, asking people to circulate the call as widely as possible to gather responses from as many primatologists as possible. This report compiles the responses received.

I received 170 questions in 32 responses, which I supplemented with personal communications and discussions at the IJP editorial board meeting in Cancun. This represents about $2 \%$ of the IPS membership at the time of the Cancun Congress (ca. 1430 members in good standing; Schapiro 2012). Respondents included individuals and research groups from the United States, Germany, Democratic Republic of Congo, Italy, France, the United Kingdom, and Canada and contributed 1-36 questions each (median 3, mode 1). The questions ranged in breadth from the speciesspecific (e.g., how to conserve a particular species in a particular National Park) to the very broad (e.g., the evolution of sociality), and in detail from a few words to pages. I also received several very positive comments on the search itself, suggesting that the search had generated interesting discussion in research groups.

J. M. Setchell ( $\square)$

Department of Anthropology, Durham University, Durham DH1 3LE, UK

e-mail: joanna.setchell@durham.ac.uk 
Below, I categorize the questions by broad theme, grouping subthemes where appropriate (one research group categorized its own questions, which helped). I reproduce most questions as they were submitted, but I have spelled out some acronyms and shortened some questions. I show cuts with [ ... ] and note any edits or rephrasing in square brackets. In some cases respondents made very specific statements from which I have extrapolated a more general question. Where questions included sub-questions, I took the decision to split or lump these based on the level of detail. In one case I combined several very similar questions from the same respondent. I note all these editorial decisions. I cut one question about elephants, but include all others. Having done this, I circulated a draft to four participants for their comments and incorporated their feedback into the final version. I present themes in descending order of the number of questions, but of course this is based on subjective categorization.

\section{How can we conserve primates in the face of global change? (46 questions)}

This was the most frequent theme by far, accounting for about a quarter of the questions received and divided into 11 subthemes.

General conservation questions:

- The primates are one of the few larger groups of vertebrates that were lucky not to lose any species over the last century. How can we ensure that we do not lose any primate taxa over the next century either?

- What is the best way to conserve primates?

- How will we ensure a future for all species of primates?

- What changes are needed in landscapes (including socioeconomic landscapes and institutions) to ensure each primate species survives into the 22nd century? If we can be clearer on this, generations of students may find it easier to contribute work that is of greatest relevance to conservation.

- What is the rate of disappearance of primate species?

- How can we coexist with primates? or How can we help primates to continue to exist?

How does global change affect primates?

- How does global change impact on primates? [rephrased as a question]

- How do we predict how primates will respond to global change (includes climate change, urbanization and rural abandonment, anthropogenic change, pollution)?

- Which species are most likely to survive (or flourish in) future climate changes?

- How will climate change affect ecosystems and species?

- Can the primates of central Africa adapt to climate warming? [I condensed three very similar questions from the same respondent into one here.]

How do habitat degradation and fragmentation affect primates?

- How do fragmentation and habitat loss affect genetic composition and gene flow in isolated populations of primates? 
- How do primates perceive the fragmented landscape and how do they move through it?

- Which behaviors observed in wild populations are the results of evolutionary processes versus the results of living in a degraded and/or fragmented environment?

- What is the effect of forest fragmentation on primates around the world?

- How permeable are different matrix types in the landscape for primates? [rephrased as a question]

- How can we conserve primates in the present, taking into account that for most primate habitat countries, the only available habitat is highly fragmented? Should we forget the long-held idea of conserving vast areas of untouched national parks and start thinking that this is no longer possible and we now have to conserve fragmented areas?

- Do pesticides modify primate habitat in Africa? [rephrased]

- Can primates adapt to the modernization of agriculture in Africa? [rephrased]

- What distinguishes species that are adaptable to disturbance from those that are not?

Questions about commensalism, coexistence, and tourism:

- How can we avoid frustrated humans slaughtering primates and avoid disease transmission between other primates and humans? [rephrased more generally than the original, and as a question rather than a statement]

- Can we adapt existing models of primate tourism to other taxa? [rephrased more generally than the original, and as a question rather than a statement]

How can we identify priorities and what strategies should we use to conserve primates?

- If limited funds and time will not allow rescue of all endangered primate populations, how do we decide which ones to prioritize?

- How do we move from a "react to" conservation strategy to a "predict and prevent" strategy?

- How can primatologists make REAL change with respect to conservation?

- Which species of primates require the most urgent conservation action to save them for the future?

- How do we determine which groups to invest conservation resources in?

How do conservation and development interact?

- What is the best way to help local communities and make positive conservation gains at the same time?

- What sort of development will help to protect primates in national parks? [rephrased as a more general question]

What do primates need to survive?

- How much variation (ecological, genetic, etc.) is required to maintain a selfsustaining population of nonhuman primates?

- In light of human encroachment, climate change, etc., what are individual species' requirements for living on a landscape? 
How do we achieve attitude change?

- How can the world population learn to protect endangered species?

- How can we convince human beings of the intrinsic value of our fellow primates?

- How can we teach humans who eat primates that primates are not acceptable in the human diet and how can we help offer alternatives?

- How do we show governments, institutions, and the public that primates matter?

Policy and politics:

- What are successful strategies to change policy?

- Do international politics favor the protection of primates?

Rehabilitation and restoration:

- How successful are rehabilitation and/or reintroduction programs?

- How can we best restore forests and the primates that depend on them?

Other questions concerning primate conservation:

- How do factors such as gender influence people's contribution to primate conservation? [rephrased more generally]

- How does poverty influence the protection of primates in Africa? [rephrased more generally]

- Where will we be in successive generations if shifting baselines continue to slide? The changes in the last 100 years have been dramatic, but is our baseline of what is acceptable changing every generation? (e.g., the shift from conservation involving protection versus community involvement)

- Do the risks of studying a new, unhabituated group outweigh the benefits of what we might learn by observing it? Can we better protect unstudied groups by instead learning more about currently studied groups?

- Are hybrid zones necessary for conservation?

\section{The evolution of sociality and social behavior (28)}

Questions in this theme fell into seven subthemes.

General questions about the evolution of sociality and socioecology:

- How and why did sociality evolve? [rephrased as a question]

- Why do so many primates live in groups?

- Why do primates have such great diversity in their social organizations? [This could be broadened to "Is the diversity of primate social organizations in fact larger (given their species diversity) than that of other major mammalian orders, and if so, why?']

- To what degree do diet and foraging and ranging behavior constrain primate sociality?

- Just how important is predation for primate social systems?

- Can one make predictions about the relative importance of phylogeny, mate access, diet, etc. on sociality in any given species of primate? 
- What factors contribute to primates' social and ecological decision making, and how does this relate to kinship and environment?

- Socioecology is founded on the premise that resource abundance and distribution are causally related to social structure, yet the mechanism for this relationship is described mostly in verbal models that are difficult to test precisely. Mathematical models exist for one category of social foraging (producerscrounger models), but these have been applied only occasionally to primates and never integrated into socioecological theory. My question: Can producerscrounger models (or any other existing mathematical framework) be used to guide future studies of socioecology by defining exactly what parameters of resources should relate, in what precise ways, to which particular aspects of social behavior?

- Are social organization and mating system responses to ecological and demographic selection pressures, and how do these differ between various primate taxa (radiations or parts of radiations)? [rephrased as a question]

- How do primates cope with ecological and social "risk" in relation to intergroup aggression, predation, etc.? [rephrased as a question]

- How can we include cross-sex interactions in the socioecological model?

Intraspecific questions:

- Are there ecological determinants of what was long considered to be "speciestypical behavior" but turned out to be specific to certain groups?

- What are the ecological correlates of and phylogenetic constraints on intraspecific variation in social organization? [rephrased as a question]

Questions about definitions:

- What factors define "social complexity" in primates and how is that complexity different from (or similar to) other animals?

- How do we measure sociality for comparative study?

- What is a species' social organization?

Comparative questions specific to some primate taxa:

- Despite rumors to the contrary, the folivore paradox is alive and well, in general (certain exceptions notwithstanding). What limits group sizes in those folivores in which scramble competition is apparently minimal? Why do some folivores show linear hierarchies whereas others do not?

- Why do female Old World primates care so much about status per se (e.g., willing to fight over status even in the absence of an immediate resource to be gained), and New World primates seem to care so little about it (rarely fight if no contestable resource is at stake)?

- Are living chimpanzees the best model for the behavior and ecology of fossil humans?

- How can one infer sociality in fossil primates?

Questions specific to fission-fusion dynamics:

- What are the opportunities created by fission-fusion dynamics?

- How is the nature of social interactions affected by differences in time spent with group members? 
- What are the challenges of fission-fusion dynamics?

Social behavior and fitness:

- How does social networking relate to feeding/disease/social bonding/infanticide in primates?

- The structure (properties) of the social network might favor some social phenomena, such as group decision-making efficiency, but also bring some disadvantages to central individuals, such as an increase in disease transmission and infections. My question is whether the social network can be selected to increase the fitness of group members and how can this social network be selected at the individual level (genetic, epigenetic, physiology, social constraints)?

- Does living with relatives promote nepotism, or vice versa? How would you test the two possibilities?

- What is the relationship between individual success and the success of the group?

And an unresolved question about mechanisms of social behavior:

- Do differences in stress hormonal responses reflect adaptations of populations to different social or ecological stressors?

\section{Brains and cognition (23)}

These questions fell into six subthemes.

Evolution of large brain size:

- How did large brains and cognitive complexity evolve in primates? What are these traits used for?

- Why are primate brains big? or Why do primates need to be intelligent?

Evolution of culture:

- Do primates have "culture"?

- What is "culture" in primates?

- Why do some primates appear to have cultural traditions, while others do not?

- Why do nonhuman primates lack cumulative culture?

- How common is the innovation of new behaviors in groups of wild primates?

Questions addressing particular cognitive abilities or indicators of brain specialization:

- How and what do primates remember?

- What are the mechanisms by which primates recognize other individuals and remember them without seeing them for long periods of time? [This fits neatly with questions about fission-fusion, elsewhere.]

- What drives the emergence of tool use in primate groups?

- Are any nonhuman primates right-handed at the species level rather than the group/population level?

- Do (some) primates have the capacity for "language"? 
- Do nonhuman primates have emotions and thoughts?

- [...] Do apes spend more or less of their time in what John Crook might call "attentional" states (Zen, meditation) and less in "intentional"?

- What information can primates convey in vocalizations? (i.e., what can primates tell each other?)

- In what ways does cognition vary across taxa and how is this related to their phylogenetic and ecological characters? [This could be expanded to include nonprimates, e.g., how does cognition in other taxa compare to that of primates?]

- How are cognitive abilities affected by the degree of fission-fusion dynamics?

Methodological questions:

- Can we come up with measures of cognition that are comparable across and within species?

- Can we build cognitive tasks that allow primates to perform at the peak of their ability?

- What do cognitive studies of captive primates tell us about the abilities of wild individuals?

Questions about neural architecture and pathology:

- How well do differences in neural architecture explain cognitive differences between humans and nonhuman primates?

- How well do differences in neural architecture explain differences in neurological and psychiatric diseases between humans and nonhuman primates?

- Do chimpanzees manifest their own type of schizophrenia?

Most, although not all, of the above are human evolution-oriented questions, interested primarily in the extent to which other primates share human cognitive abilities. However, one respondent noted that:

- Much cognitive work focuses on "What makes humans unique?" Turning the question around to "What is unique about primate cognition?" could open up our understanding of primates.

\section{Evolutionary biology (20)}

This broad theme fell into seven subthemes.

Biogeography:

- How did New World primates get there?

- Why aren't nonhuman apes everywhere that monkeys are? (i.e., what different niches do they fill?)

Genotype $\times$ environment interactions and the relationship between genotype and phenotype:

- How do individual experience and genetic and epigenetic inheritance work together to shape individual development? 
- What is the mapping between the genetic information (genotype) and morphological/behavioral/anatomical variation (phenotype) in primates? (i.e., phenomics?)

- What is the link between genes and behavior in natural populations and organisms? [rephrased as a question]

The implications of new genetic methods (which may or may not fit neatly in this theme, and do not necessarily relate only to primates):

- What are the implications of having the full order of primates genome-mapped within the decade?

- What do whole genomes mean for species concepts?

- What are the causes and fitness consequences of differential gene regulation in wild primate populations? [reworded]

One respondent suggested several questions about hybridization, including:

- How do ecological and behavioral plasticity in hybrids relate to speciation? [reworded]

- Which primate species can hybridize?

One group contributed several sub-questions about phenotypic plasticity that overlap with other themes:

- What are the extents and limits of behavioral and social flexibility (phenotypic plasticity)?

- How is this influenced by scale (space/time/individual/population/species/genus)?

- How can we surpass the sample size issue? (i.e., the more studies or longer the study the more flexibility we document?)

- What are the conservation implications of this flexibility?

- What processes lead to the evolution of this flexibility?

- What role does social learning play in this flexibility?

Sexual selection:

- When is dominance associated with reproductive success?

- Sexual selection has recently been implicated to be a major determinant of social organization, but it has been difficult to quantify. What role does sexual selection play in determining primate behavior, social organization, or morphology?

- How does a modern understanding of sex roles affect the way we study sexual selection in primates?

- What factors contribute to variation in female reproductive success?

- Why do lemurs differ from the general rules established for anthropoids (e.g., sexual monomorphism with polygyny)?

Other questions that relate to primate evolutionary biology:

- What can strepsirrhines and tarsiers tell us about the evolution of the primate lineage?

- How does living in proximity to human groups (and therefore human-based resources, such as trash) affect the survival of certain groups and the emergence/persistence of certain behaviors? 
- What are the implications of artificial selection (domestication) of captive and wild primate populations? [rephrased as a question]

- What's the difference between a monkey and an ape? [I followed this one up, and it relates to questions posed by other people to primatologists, rather than by primatologists themselves.]

- How important is olfaction to primates?

- How many species of primate are there?

\section{Our interactions with other primates and ethics (12)}

The conservation theme clearly involves both our interactions with other primates and ethical issues. Other questions on this general theme fell into six subthemes.

How do we interact with other primates?

- What is the nature of the relationship between the personnel (principle investigators, vets, animal care technicians, etc.) in nonhuman primate research laboratories and the nonhuman primates used for research? Has it changed over the years? What kinds of effects does it have on both the staff and study subjects?

How can we interact with other primates?

- How can human and nonhuman primates best communicate with each other?

How should we interact with other primates?

- Can it be considered ethical to keep primates in captivity?

- Should primates be kept as pets?

- Should primates be used in medical research?

- Should primates be granted "human" rights?

- How would granting human rights change our relationship with nonhuman primates?

One respondent wrote at some length about the need for nonhuman primates in medical research, and the damaging effect of cumbersome control systems on such research. This wasn't phrased as a question, but does relate to the questions of when, where, and how medical research is conducted on nonhuman primates. We might ask:

- What is the definition of "necessary" medical research using nonhuman primates?

Linked to this are questions of captive welfare:

- Which stressors in a captive environment can be effectively mitigated (e.g., with appropriate enrichment and diet)?

- How do primates feel about living in captivity? (e.g., are they aware that they are in captivity?)

Trade in nonhuman primates for medical research. Two respondents referred to this. One asked:

- Why aren't primatologists taking action about the global trade in macaques? [I shortened this considerably.] 
The other stated that conservation, although important, is too frequently uncritically emotional and monothematic and that IPS should applaud the thoughtful and humane development of trade in non-endangered primates, support responsible control of transportation, and encourage CITES (Convention on International Trade in Endangered Species) to remove non-endangered populations from the endangered species list. From this I derived the following:

- How should we treat non-endangered primates? Can they be viewed as a resource? If so, how should exploitation of that resource be regulated?

\section{What do primates eat? (11)}

Questions about nutritional/feeding ecology fell into three subthemes.

One lab contributed several related questions/sub-questions centered on "what is food"?

- What is food? Primatologists often define food arbitrarily (e.g., the 10 most commonly eaten items), but how is this defined on a monthly scale or annual scale?

- What is a fallback food - just lower on the preference scale? Are there consistent types of fallback foods (e.g., seeds, mature leaves) or is this variable between periods and populations? How does this inform the fossil record?

- How do we define food quality?

- When is a plant a medicine?

- What is the role of secondary compounds/phytoestrogens?

- What is the role of social learning in determining foods? [linking to the cognition theme]

Two other questions also concerned secondary compounds:

- To what extent are fruit-eating primates limited in how they feed in particular trees or on particular days by the inability to handle secondary compounds in fruits?

- How do primates balance nutrients and toxins in relation to food availability?

Other questions related to feeding ecology:

- What is the appropriate spatial and temporal scale needed to address the question of what is a species diet?

- What is the relationship of digestive physiology and nutrient value of foods to gut anatomy in primates? [reworded as a question]

- What is a primate's niche? [rephrased as a question]

\section{Other aspects of ecology (8)}

These fell into four broad areas.

Ecosystems and community ecology:

- Are primates essential to ecosystems? 
- What role do primates play in ecosystem maintenance?

- How do primates coexist in the same habitat? [rephrased as a question]

What regulates populations?

- What limits populations?

- What regulates primate populations? Food resources (fruits, leaves, flowers, insects) or ecological interactions (predation, competition, parasites): Testing the up-down and bottom-up hypothesis.

- Are primates predators or prey?

Other ecological questions:

- What factors influence primate movement patterns and what abilities must they possess to produce different types of movement patterns?

- How far do primates stray out of their home ranges?

\section{Life history (5)}

Five questions fell in the broad area of life history:

- What are the ecological/physiological correlates of the life history and demography of primates? [rephrased as a question. This links to ecology and physiology, earlier]

- How does the biology of aging (e.g., anatomical, behavioral, physiological, and cognitive changes) differ among primates, and why?

- What is the significance of play in ontogeny?

- Is body size a sufficient proxy for life-history variation among primate taxa for the purposes of comparative studies, or do we need to focus on more precise (but more difficult) variables such as mortality or agespecific fecundity?

- How is neural system development influenced by the environment (neuroanthropology)?

\section{Disease ecology (3)}

These included two questions about zoonoses:

- What role do primates have in zoonoses?

- What are the implications of disease transmission among and within species, for conservation and zoonotic disease ecology? [reworded]

And one about the influence of parasites on primates:

- How sensitive are long-lived primates to the effects of parasite infestation? Is there any evidence that chronic parasite infestations have demographic consequences? Are these restricted to certain kinds of individuals (juveniles, subordinates) or times (low food abundance)? 


\section{Applied (medical) research (2)}

This topic included one broad and one specific question:

- What are the prospects for the use of nonhuman primates in applied research in different countries around the world?

- How good of a model for neuropsychiatric disorders are nonhuman primates?

\section{How do we promote primatology? (2)}

The question of how we promote primatology arose either twice or five times, depending on how we treat sub-questions:

- How do we "promote" primatology to:

- granting agencies

- nonhost countries

- host countries (urban and rural)

- policy makers?

- How may we (and should we) develop and empower primatologists in government, nongovernmental organizations, and academia in the developing world?

\section{Miscellaneous (10)}

Other questions that fell outside the preceding themes were:

- Who have been the most important contributors to primatology?

- What's the most "successful" nonhuman primate?

- What characterizes resilience at the individual level, and what characteristics of behavior and physiology support resilience? [This is relevant to conservation, but also has broader relevance.]

- How can interdisciplinary projects be encouraged?

- If primates could talk, what would they talk about?

- How comparable are studies (e.g., physiological or behavioral) done in the lab to the results that would be obtained in the wild? Can we make the conditions of laboratory studies more similar to those in the wild?

- More research is needed into the behavior, ecology, genetics, and cognition of species that have not been as thoroughly studied as other species to date (such as bonobos, colugos [primates' closest outgroup], etc. [This might be rephrased as "which species should we be focusing on"?]

- How best to reassess our present efforts and promulgate new methods to collate, assess, and distribute primate information? 


\section{Initial Conclusions and Next Steps}

The response rate, which is low even for Internet surveys, may reflect a lack of reminders to submit questions, a lack of perceived relevance, lack of incentive to contribute (for comparison, the IPS research grant competition received 80 applications this year), or simply time pressure. Alternatively, as one contributor pointed out, people may be reluctant to share their research ideas. This seems sad - our science will advance more slowly if we do not discuss ideas openly. There is no danger that we will run out of questions, as this list demonstrates.

As Charlie Janson commented (via e-mail, 2013), the questions can be divided into two major types: policy and research. Both types are important, but they call for very distinct sets of skills and approaches. Not surprisingly, most of the conservation questions fall into the former category, while many of the remaining questions are in the latter. Arguably, the policy questions, especially those with respect to conservation, require multidisciplinary teams, often including non-scientists. The unspoken question behind many of the policy questions is "How can scientists help?" In other words, what are the major scientific gaps, the answers to which would help shape policy concerning conservation? Some of these are implicit in the more specific questions concerning conservation, including climate change, habitat degradation, tourism, and development. In addition, some of the scientific questions may not be limited to the field of primatology (e.g., what models of social change work best to promote local conservation, and do the applicable models differ predictably in different countries or regions?).

The focus on conservation issues is unsurprising, given the status of our study taxa. It is also important that we think about this - as highlighted by a respondent, doing so will help us identify and concentrate on the work that has greatest relevance to conserving primates. The next largest foci, on the evolution of sociality and on brains and cognition, are also unsurprising as these are the unusual aspects of primates when compared with many other species. Other themes represent broader questions in evolutionary biology and ecology and might equally be asked by researchers studying other taxonomic groups, such as ungulates, cetaceans, or rodents. Questions of ethics and welfare fall somewhere between the two, depending on whether you see them as primate-specific due to phylogenetic relatedness (phylogenetic luck?), or as more general.

Some of the more strictly research questions divide into proximate and ultimate, while others address both. Some methods, such as noninvasive hormone assays, which appear in the list, and remote monitoring, which does not, transcend specific research questions and can be applied equally to conservation issues and the causes or consequences of sociality. These technologies have contributed new insights to our science, and will continue to do so as technology develops. Another major integrative theme that transcends several research areas is the question of how genes interact with environment to "create" individual responses to ecological and social circumstances that together "create" what we call social structure and organization.

The next question to consider is whether entire themes are missing. A glance at my bookshelves and reference collection and at the contents of journals suggests 
primatologists work on many topics in addition to those listed in the preceding text. For example, as someone who attends the Göttinger Freilandtage conferences, I was surprised to see so few questions relating to sexual selection, and only one mention of infanticide. As Charlie Janson suggests (via e-mail, 2013), this may be because many feel that topics tackled by such meetings (and the subsequent edited volumes) have been treated so recently and thoroughly that they do not qualify as unanswered questions. Nevertheless, plenty of responses to the survey fell into the "long-standing but very hard to resolve" problems category.

Some questions in this list have already provoked reactions from readers. This is a good sign. I would be delighted to receive responses to this editorial, either personally via e-mail, or as commentaries submitted to IJP. These could be general comments, or focus on specific, or missing, themes. My ultimate aim is to expand this list into a series of reviews for the International Journal of Primatology, highlighting the questions that primatology should be seeking to answer; the "opportunities to be exploited" (Siegfried 2005). For the moment, I'll end with two thought-provoking comments from participants:

- Which hypotheses should we now junk? (Charlie Janson, pers. comm. at IPS 2012)

- Asking what is NEW would be a good idea since I see a lot of the same old questions there that we have looked at for decades (Colin Chapman, via e-mail 2013).

\begin{abstract}
Acknowledgments I am very grateful to all the survey respondents: Filippo Aureli; Lucy Bates; Susan Cachel; Colin Chapman and his lab, including Mike Wasserman, Jan Gogarten, and Sarah Turner; David Chivers; Ben Coleman; Elena Cunningham; Thomas Defler; Frank Ervin; Dorothy Fragaszy; John Fellowes; Lyn Isbell; Charlie Janson; Patrick J Gannon; Thallita Grande; Rachel Kendal; Victoria Martindale ; Blake Morton; Laban Mvukiyehe; Marc Myers; Liz Renner and other primatologists in the Department of Anthropology and the Center for the Advanced Study of Hominid Paleobiology at George Washington University (including faculty, postdocs, and graduate students); Jess Rothman and her lab, including Margaret Bryer, Caley Johnson, and Bianca Shannon; Christoph Schwitzer; Stuart Semple; Cédric Sueur; Dr. Natalie T. Uomini; Lina Maria Valencia ; Augusto Vitale; J. C. Voltolini; Barb Welker; and Anja Widdig. I am particularly grateful to Colin Chapman, Charlie Janson, Rachel Kendal, and Jess Rothman for commenting on an earlier draft of this editorial, and to many primatologists for conversations on the topic at IPS 2012.
\end{abstract}

\title{
References
}

Giles, J. (2011). Social science lines up its biggest challenges. Nature, 470, 18-19.

Kennedy, D., \& Norman, C. (2005). What don't we know? Science, 309, 75.

Schapiro, S. (2012). Trea\$ury note\$. IPS Bulletin, 38, 6-7.

Setchell, J. M. (2012). On editing the International Journal of Primatology. International Journal of Primatology, 33, 1-9.

Siegfried, T. (2005). In praise of hard questions. Science, 309, 76-77.

Sutherland, W. J., Adams, W. M., Aronson, R. B., Aveling, R., Blackburn, T. M., Broad, S., Ceballos, G., Côté, I. M., Cowling, R. M., et al. (2009). One hundred questions of importance to the conservation of global biological diversity. Conservation Biology, 23, 557-567.

Sutherland, W. J., Aveling, R., Bennun, L., Chapman, E., Clout, M., Côté, I. M., Depledge, M. H., Dicks, L. V., Dobson, A. P., et al. (2012). A horizon scan of global conservation issues for 2012. Trends in Ecology \& Evolution, 27, 12-18. 
Sutherland, W. J., Bardsley, S., Bennun, L., Clout, M., Côté, I. M., Depledge, M. H., Dicks, L. V., Dobson, A. P., Fellman, L., et al. (2011). A horizon scan of global sonservation issues for 2011. Trends in Ecology and Evolution, 26, 10-16.

Sutherland, W. J., Bardsley, S., Clout, M., Depledge, M. H., Dicks, L. V., Fellman, L., Fleishman, E., Gibbons, D. W., Keim, B., et al. (2013a). A horizon scan of global conservation issues for 2013. Trends in Ecology and Evolution, 28, 16-22.

Sutherland, W. J., Clout, M., Côté, I. M., Daszak, P., Depledge, M. H., Fellman, L., Fleishman, E., Garthwaite, R., Gibbons, D. W., et al. (2010). A horizon scan of global conservation issues for 2010. Trends in Ecology \& Evolution, 25, 1-7.

Sutherland, W. J., Freckleton, R. P., Godfray, H. C. J., Beissinger, S. R., Benton, T., Cameron, D. D., Carmel, Y., David, A., Coulson, T., et al. (2013b). Identification of 100 fundamental ecological questions. Journal of Ecology, 101, 58-67. 